Recepción: 20 / 04 / 2017

Aceptación: 20 / 05 / 2017

Publicación: 15 / 07 / 2017

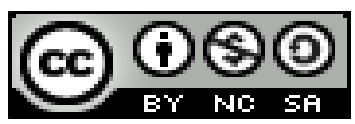

Ciencias de la Educación

Artículo Científico

\title{
Estrategias didácticas en el aprendizaje del inglés técnico
}

\author{
Didactic strategies in the learning of technical English
}

Estratégias de ensino na aprendizagem de Inglês técnico

\author{
Ana M. Herrera-Espinoza ${ }^{\mathrm{I}}$ \\ aherrera@uagraria.edu.ec \\ Jenny A. Ortiz-Zambrano II \\ jenny.ortizz@ug.edu.ec \\ Karen M. Mite-Baidal III \\ kamite@uagraria.edu.ec \\ Evelyn T. Solís-Avilés IV \\ esolis@ uagraria.edu.ec
}

Correspondencia: aherrera@ugraria.edu.ec

Docente, Universidad Agraria del Ecuador, Guayaquil, Ecuador. Docente, Universidad de Guayaquil, Guayaquil, Ecuador.

Docente, Universidad Agraria del Ecuador, Guayaquil, Ecuador.

Docente, Universidad Agraria del Ecuador, Guayaquil, Ecuador. 


\section{Resumen}

La importancia de esta investigación está ligada a elevar el nivel académico de la Carrera de Licenciatura en Sistemas de Información de la Facultad de Ingeniería Industrial de la Universidad de Guayaquil, pues se definen pautas para mejorar el inter aprendizaje del Inglés Técnico, mediante el diseño de una Guía Didáctica, lo que se reflejaría en el mejoramiento de la formación académica y profesional de quienes se educan en dicho centro universitario; siendo por tanto, los estudiantes, y docentes en general los beneficiarios de este trabajo. En lo metodológico es una investigación cuantitativa - cualitativa; la modalidad corresponde a un Proyecto Factible porque cuenta con el apoyo de autoridades, docentes y estudiantes de dicho centro de educación superior, siendo también una investigación de Campo, porque se llevó a cabo en el mismo lugar de los acontecimientos, además es una investigación bibliográfica, pues se consultó información en una variedad de libros, lo cual sirvió de base para la elaboración del marco teórico, así como para el desarrollo de los contenidos de la guía didáctica propuesta; además, las técnicas utilizadas fueron observación, encuesta que fue dirigida a autoridades, docentes y estudiantes.

Palabras Claves: Licenciatura; académico; aprendizaje; guía; didáctico. 


\section{Abstract}

The importance of this research is linked to raising the academic level of the Degree in Information Systems of the Faculty of Industrial Engineering of the University of Guayaquil, as guidelines are defined to improve the inter learning of Technical English through the design of A Didactic Guide, which would be reflected in the improvement of the academic and professional education of those who are educated in said university center; Being therefore, the students, and teachers in general the beneficiaries of this work. In the methodological is a quantitative - qualitative research; The modality corresponds to a Feasible Project because it has the support of authorities, teachers and students of this higher education center, being also a Field investigation, because it was carried out in the same place of events, in addition it is a bibliographical research, Since information was consulted in a variety of books, which served as a basis for the development of the theoretical framework, as well as for the development of the contents of the proposed didactic guide; In addition, the techniques used were observation, a survey that was addressed to authorities, teachers and students.

Key Words: Bachelor; academic; learning; guide; didactic. 
Ana M. Herrera-Espinoza; Jenny A. Ortiz-Zambrano; Karen M. Mite-Baidal; Evelyn T. Solís-Avilés

\section{Resumo}

A importância da pesquisa está ligada ao elevador do nível académico da Carrera de Licenciatura em Sistemas de Informação da Faculdade de Engenharia Industrial da Universidade de Guayaquil, pois você pode definir pacotes para melhorar a interação do inglês Técnico, mediante o design de Una Guía Didáctica, que se reflete no melhoramento da formação académica e profissional de quem é educado em centro; Sendo por tanto, os estudantes e docentes em geral dos beneficiários deste trabalho. En lo metodológico é uma pesquisa cuantitativa - cualitativa; A modalidade corresponde a un Projeto Factible porque conta com o apoio de autoridades, docentes e estudantes de centro de educação superior, também é uma investigação de Campo, porque se levou um cabo no mesmo lugar de acontecimentos, além de uma pesquisa bibliográfica $\mathrm{E}$, assim, para o desenvolvimento dos conteúdos da candidatura didática proposta; Além disso, as técnicas utilizadas foram consultadas, encuesta que foi dirigida a autoridades, docentes e estudiantes.

Palavras-chave: Graduado; académico; à aprendizagem; guia; didactico. 


\section{Introducción.}

Es necesario que los Institutos de Educación Superior garanticen calidad profesional, científica y humanística, tanto en sus docentes como en sus estudiantes, y que acredite que las futuras generaciones de profesionales que se desempeñen con excelencia y competitividad en su entorno profesional. Contribuyendo a la aplicación de las Estrategias Didácticas en el aprendizaje del Inglés Técnico, en los estudiantes de la Carrera de Licenciatura en Sistemas de Información de la Facultad de Ingeniería Industrial de la Universidad de Guayaquil, tiene como finalidad desarrollar las habilidades lingüísticas.

Además, permitirá conocer las principales causas del bajo rendimiento en los estudiantes de la Carrera, tales como: los diferentes niveles del idioma inglés, la ausencia de los recursos didácticos, la carga horaria, y el desconocimiento de metodologías y técnicas adecuadas, por lo que se propone desarrollar una Guía Didáctica en la enseñanza del Inglés Técnico, que permita un manejo adecuado del idioma inglés como medio de comunicación, compresión e interpretación.

\section{Materiales y métodos (Metodología).}

Se midió una información cuantificable sobre las deficiencias en el proceso de enseñanza aprendizaje del inglés técnico; que mide la frecuencia de la información recogida y se muestra cómo se presenta el fenómeno.

En la presente investigación se utilizó el análisis para lograr una comprensión más detallada y profunda, las técnicas utilizadas permitieron comprender el fenómeno, responder interrogantes, alcanzar las motivaciones, percepciones y orientaciones valóricas de los sujetos y la interpretación del fenómeno social 
Para el presente estudio la población estuvo conformada por: 2 autoridades, Director y Coordinador, 2 docentes de la materia de inglés y 156 estudiantes que conforman la Carrera de Licenciatura en Sistemas de Información de la Facultad de Ingeniería Industrial de la Universidad de Guayaquil. Por tanto, la población involucrada en el presente estudio, está conformada por un total de 160 personas.

Tabla 1: Operacionalización de Variables

\begin{tabular}{|c|c|c|}
\hline Variables & Dimensiones & Indicadores \\
\hline \multirow{2}{*}{$\begin{array}{l}\text { Independiente: } \\
\text { Estrategias Didácticas }\end{array}$} & Estrategias Didácticas & $\begin{array}{ll}\text { - } & \text { Definición } \\
\text { Importancia }\end{array}$ \\
\hline & Investigación & $\begin{array}{l}\text { Observación } \\
\text { Encuesta }\end{array}$ \\
\hline \multirow{2}{*}{$\begin{array}{l}\text { Dependiente: } \\
\text { Aprendizaje del Inglés Técnico }\end{array}$} & Aprendizaje & $\begin{array}{ll}\text { Definición } \\
\text { Tipos }\end{array}$ \\
\hline & Inglés Técnico & $\begin{array}{ll}\text { : } & \text { Métodos } \\
\text { - } & \text { Estricnicas } \\
& \text { Estrategias }\end{array}$ \\
\hline \multirow{3}{*}{$\begin{array}{l}\text { Dependiente: } \\
\text { Propuesta de una guía didáctica }\end{array}$} & Diseño & $\begin{array}{ll}: & \text { Introducción } \\
: & \text { Estrategias } \\
: & \text { Objetivos } \\
\text { Proceso } & \text { Recomendaciones }\end{array}$ \\
\hline & Aspectos & $\begin{array}{ll}\text { - } & \text { Filosófico } \\
\text { - } & \text { Psicológico } \\
\text { - } & \text { Educativo } \\
\text { Sociológico }\end{array}$ \\
\hline & Validación & - Instrumentos \\
\hline
\end{tabular}

Fuente: Elaboración Propia. 


\section{Resultados}

- Un 75\% de los encuestados alegó estar totalmente de acuerdo y el otro $25 \%$ consideró estar de acuerdo, lo que evidenció que el 100\% consideró que el Inglés Técnico se aplica en el mundo laboral y social.

- Un 50\% de los encuestados expresó estar totalmente de acuerdo y el otro 50\% manifestó estar de acuerdo, totalizando un $100 \%$ que consideró que el inglés es un idioma utilizado en la comunicación oral y escrita.

- Todas las autoridades y docentes encuestados aseguraron estar totalmente de acuerdo en que es necesario dejar de considerar como una opción el aprendizaje del Inglés Técnico.

- Todas las autoridades y docentes encuestados dijeron estar totalmente de acuerdo en que el aprendizaje del Inglés Técnico debe ser un requisito en la formación personal, académica y profesional.

- El $100 \%$ de los encuestados opinó estar totalmente de acuerdo al señalar que la enseñanza del Inglés Técnico no debe basarse en ejercicios de memorización y repetición.

- El 100\% de autoridades y docentes encuestados manifestó estar totalmente de acuerdo en que las estrategias didácticas facilitan el aprendizaje del Inglés Técnico.

- Todas las autoridades y docentes encuestados consideraron que es necesario diagnosticar y evaluar periódicamente la enseñanza del Inglés Técnico en la Carrera de Licenciatura en Sistemas de la Información de la Facultad de Ingeniería Industrial.

- El 100\% de los encuestados consideró que los docentes de la Carrera de Licenciatura en Sistemas de la Información deben adoptar estrategias didácticas para que la enseñanza aprendizaje del Inglés Técnico sea dinámico y motivador. 
Ana M. Herrera-Espinoza; Jenny A. Ortiz-Zambrano; Karen M. Mite-Baidal; Evelyn T. Solís-Avilés

- El 100\% de los encuestados consideró que es necesaria la elaboración de una guía de estrategias didácticas para la enseñanza - aprendizaje del Inglés Técnico en la Carrera de Licenciatura en Sistemas de la Información de la Facultad de Ingeniería Industrial.

- Todas las autoridades y docentes encuestados indicaron estar totalmente de acuerdo en que el diseño de una guía de estrategias didácticas debe responder a las necesidades de los cambios en la enseñanza - aprendizaje del Inglés Técnico.

- El $100 \%$ de los encuestados estuvo totalmente de acuerdo en que el rendimiento académico de los estudiantes se favorece con el diseño de una guía de estrategias didácticas para la enseñanza - aprendizaje del Inglés Técnico.

- El 50\% de las autoridades y docentes encuestados afirmó tener de 11 a 15 años de ejercicio profesional y el otro 50\% indicó tener de 16 a 20 años de ejercicio profesional en la cátedra universitaria.

- El 50\% de los encuestados indicaron tener nivel básico de conocimientos adquiridos en estrategias didácticas para la enseñanza - aprendizaje del Inglés Técnico y el otro 50\% aseveraron no tener ninguno.

- Todos los encuestados indicaron que no han recibido algún curso de capacitación sobre estrategias didácticas para la enseñanza - aprendizaje del Inglés Técnico en los últimos cinco años.

- De los resultados se determina que un $25 \%$ de los estudiantes encuestados tiene una edad entre 17 a 25 años, un 5\% de 26 a 35 años, un $40 \%$ de 36 a 45 años, un $25 \%$ de 46 a 55 años y un $5 \%$ de 56 a 65 años.

- Un 40\% de los encuestados manifestó estar totalmente de acuerdo, otro 55\% alegó estar de acuerdo, lo que evidencia que el 95\% consideró que el uso de estrategias didácticas en la 
enseñanza - aprendizaje contribuyen a la formación integral de los estudiantes; frente a un $5 \%$ que se mostró en desacuerdo.

- Un $65 \%$ de los encuestados sostuvo estar totalmente de acuerdo y un 30\% indicó estar de acuerdo, totalizando el $95 \%$ que consideró que los docentes deben utilizar estrategias didácticas que permitan crear condiciones favorables para el aprendizaje, frente a un 5\% que se mostró totalmente en desacuerdo.

- Un $45 \%$ de los encuestados dijo estar totalmente de acuerdo y otro $45 \%$ se consideró de acuerdo, determinándose que el 90\% consideró que el aprendizaje del Inglés Técnico debe ser un requisito en la formación personal, académica y profesional, frente a un $10 \%$ que se mostró en desacuerdo.

- Un $80 \%$ de los encuestados consideró que es necesario diagnosticar y evaluar periódicamente la enseñanza del Inglés Técnico en la Carrera de Licenciatura en Sistemas de la Información de la Facultad de Ingeniería Industrial, frente a un 20\% que afirmó lo contrario.

- Un $90 \%$ de los encuestados alegó estar totalmente de acuerdo y de acuerdo en que la implementación de una guía de estrategias didácticas en la enseñanza - aprendizaje del Inglés Técnico estimula el desarrollo de habilidades lingüísticas de los futuros profesionales; frente a un $10 \%$ que se mostró en desacuerdo y totalmente en desacuerdo.

\section{Conclusiones}

- El Inglés Técnico es un idioma universal que se utiliza en la comunicación oral y escrita, tanto en el mundo de los negocios como en al ámbito social, así como en la educación, la ciencia, la política, la cultura, razón por la cual su enseñanza no debe basarse únicamente en simples ejercicios de memorización y repetición. 
Ana M. Herrera-Espinoza; Jenny A. Ortiz-Zambrano; Karen M. Mite-Baidal; Evelyn T. Solís-Avilés

- Las estrategias didácticas estimulan la motivación por el aprendizaje del Inglés Técnico, lo cual incide en un mejor rendimiento académico de los estudiantes del Cuarto nivel de la Carrerade Licenciatura en Sistemas de Información.

- Los docentes hacen uso de las estrategias didácticas en la enseñanza del Inglés Técnico, por lo cual no se desarrollan en los futuros profesionales habilidades en la expresión oral y escrita.

- Es imprescindible la elaboración de una Guía Didáctica para mejorar el proceso de enseñanza - aprendizaje del Inglés Técnico, ya que desarrollará habilidades lingüísticas en la expresión oral y escrita de los futuros profesionales, lo cual conlleva a elevar el nivel académico de dicha carrera.

\section{Recomendaciones}

- Las autoridades de la Carrera de Licenciatura en Sistemas de Información de la Facultad de Ingeniería Industrial de la Universidad de Guayaquil deben fortalecer la enseñanza del Inglés Técnico transformándola en un requisito indispensable en la formación académica y profesional de los futuros egresados.

- Los docentes del área de Inglés Técnico deben capacitarse en el uso de estrategias didácticas para transformar la práctica educativa en un proceso dinámico y motivador. debiendo adoptar las estrategias que se proponen en la Guía para que la enseñanza del Inglés Técnico se desarrolle de manera innovadora.

- Los estudiantes deben poner todo su empeño, motivación e interés por aprender una segunda lengua, como es el Inglés Técnico, el cual les ayudará en la comunicación y expresión oral y 
escrita en el campo profesional y laboral, en donde deberán poner en práctica sus capacidades y desempeños.

\section{Bibliografía}

“Constitución de la República del Ecuador" (2008) Editorial Corporación de Estudios y Publicaciones. Quito - Ecuador.

“Ley Orgánica de Educación Superior” (2014) Corporación de Estudios y Publicaciones. Quito - Ecuador. Andino, Patricio (2005) “El Proyecto de Grado”. Teoría y Gráficos. P. H. Ediciones. Quito - Ecuador.

Bernardo Carrasco, J. '(2005) “Cómo aprender mejor”. Estrategias de aprendizajes. Editorial Rialp. Madrid.

Bolívar, Martha y otros (2002) "Estrategias Pedagógicas para Encuentros”. Editorial Paulina. BogotáColombia.

Chadwick, Clifton B. (2008) "Estrategias Cognitivas y Afectivas de Aprendizaje”. Revista Latinoamericana de Psicología. Santiago de Chile.

Chastain, K. (1992) “Developing second-language skills: theory and practice”. Harcourt Brace Jovanovich. New York.

Cooper, C. (1975) "Measuring growth in writing”. English Journal.

Díaz Barriga, Frida y otros (2006) "Estrategias Docentes para un Aprendizaje Significativo”. Editorial MC Graw Hill. México.

Dudley, Evans T.; Strevens, John M. (2008) "Developments in English for Specific Purposes. A multidisciplinary approach". Cambridge University Press. Cambridge.

Emig, J. (1983) "The web of meaning: essays on writing, teaching, learning and thinking”. Boynton/Cook Publishers, Inc. New Jersey.

Freeman Yvonne S. y Freeman David E. (2007) "Teaching reading and writing in Spanish and English in bilingual and dual language classrooms". Heinemann. USA.

Gaudini, C. (1981) "Teaching composition in the foreign language curriculum". In Language in Education: Theory and Practice Series, No 43. Center for Applied Linguistics. Washington, DC.

Hernández S., Roberto y otros (2010) “Metodología de la Investigación”. Editorial Mc. Graw Hill. México.

Izquierdo Arellano, Enrique (2000) “Didáctica y Aprendizaje Grupal”. Gráficas Lizzette. Loja - Ecuador.

Jones, F., Palincsar, A, (2005) “Estrategias para enseñar a aprender”. Editorial AIQUE. Buenos Aires.

Justicia, F. y Cano, F. (2004) "Los procesos y las Estrategias de Aprendizaje”. EUB. Barcelona. 
Ana M. Herrera-Espinoza; Jenny A. Ortiz-Zambrano; Karen M. Mite-Baidal; Evelyn T. Solís-Avilés

Krashen, S. (2004) "Writing, research, theory, and applications”.Pergamon Press. Oxford.

Lewis, Michael (1985) "Practical techniques for language Teaching Language”. Teaching Publications. U.S.A

Monereo, Carles (2004) “Estrategias de enseñanza y aprendizaje”. Formación del profesorado y aplicación en la escuela. Editorial Grao. Barcelona.

Nisbet, J. Y Shucksmith, J. (2009) “Estrategias de Aprendizaje”. Editorial Santillana Siglo XXI. Madrid.

Noan Chomsky (1975) “Reflections on language”. Pantheon Books. New York.

Omaggio, A. (1993) “Teaching language in contex”. Segunda edición. Heinle \& Heinle. New York.

Patton, Michael Quinn (2006) “Qualitative research \& Evaluation Methods”. Sage Publications. Newbury Park - California - USA.

Pazmiño Cruzzatti, Iván (2008) "Investigación Científica" Tiempo de Investigar. Dimaxi. Quito Ecuador.

RAIMES A. (1983) “Techniques in teaching writing”. Oxford University Press.Oxford:

Rivers, W.M. (1975) “A practical guide to the teaching of French”. Oxford University Press. New York.

Sanchez Escobar, Ángel F. (1999) "Experience writing. A methodological approach for teaching composition to students of English as a second language". Universidad de Sevilla, Facultad de Ciencias de la Educación: Grupo de Investigación de "Lengua Española Aplicada a la Enseñanza".

Sánchez Escobar, Ángel F. (2004) “La expresión escrita en la enseñanza del Inglés”. Universidad de Sevilla.

Sarramona, Jaime (2000) "Cómo Aplicar Estrategias de Enseñanza". Editorial C.E.A.C. Barcelona España.

Silva, T. (1990) "Second language Composition Instruction: Developments, Issues, and Directions in ESL". En Kroll 1990.

Yépez Aldáz, Edison (2005) “Guía para la Elaboración del Proyecto de Trabajo de Grado”. Universidad de Guayaquil.

Zammel, V. M. (1987) “Writing: the Process if Discovering Meaning”. En Long y Richards. 